\title{
Measuring splash-dispersal of a major wheat pathogen in the field
}

\section{Article}

Published Version

Creative Commons: Attribution 4.0 (CC-BY)

Open access

Karisto, P., Suffert, F. and Mikaberidze, A. (2022) Measuring splash-dispersal of a major wheat pathogen in the field. PhytoFrontiers, 2 (1). pp. 1-83. ISSN 2690-5442 doi: https://doi.org/10.1094/phytofr-05-21-0039-r Available at https://centaur.reading.ac.uk/99365/

It is advisable to refer to the publisher's version if you intend to cite from the work. See Guidance on citing.

To link to this article DOI: http://dx.doi.org/10.1094/phytofr-05-21-0039-r

Publisher: Scientific Societies

All outputs in CentAUR are protected by Intellectual Property Rights law, including copyright law. Copyright and IPR is retained by the creators or other copyright holders. Terms and conditions for use of this material are defined in the End User Agreement.

\section{www.reading.ac.uk/centaur}

\section{CentAUR}

Central Archive at the University of Reading

Reading's research outputs online 


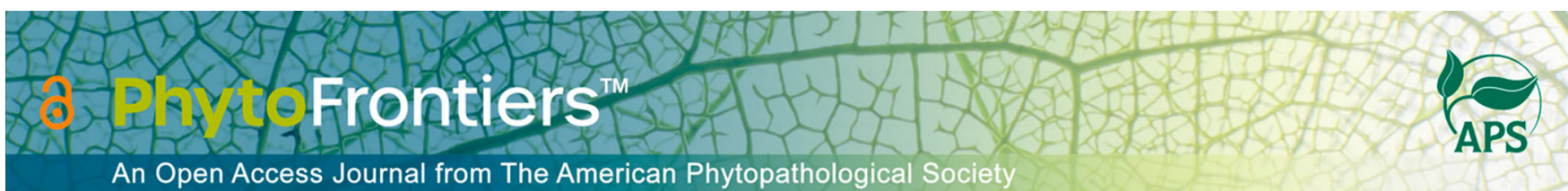

\title{
Research
}

\section{Measuring Splash Dispersal of a Major Wheat Pathogen in the Field}

\author{
Petteri Karisto $^{1,+}$ (i) | Frédéric Suffert ${ }^{2}$ (i) $\mid$ Alexey Mikaberidze ${ }^{1}$ |
}

${ }^{1}$ Plant Pathology Group, Institute of Integrative Biology, ETH Zurich, Zurich, Switzerland

2 Université Paris-Saclay, INRAE, AgroParisTech, UMR BIOGER, 78850 Thiverval-Grignon,

France

† Corresponding author: P. Karisto; petteri.karisto@luke.fi

Accepted for publication 6 July 2021.
Current address of P. Karisto: Plant Health, Natural Resources Institute Finland (Luke), Jokioinen, Finland.

Current address of A. Mikaberidze: School of Agriculture, Policy and Development, University of Reading, Whiteknights, Reading, U.K.

\section{Funding}

PK and AM gratefully acknowledge financial support from the Swiss National Science

Foundation through the Ambizione grant PZ00P3_161453.

$e-X$ tra: Supplementary materials are available online.

The author(s) declare no conflict of interest.

\begin{abstract}
Capacity for dispersal is a fundamental fitness component of plant pathogens. Characterization of plant pathogen dispersal is important for understanding how pathogen populations change in time and space. We devised a systematic approach to measure and analyze rain splash-driven dispersal of plant pathogens in field conditions, using the major fungal wheat pathogen Zymoseptoria tritici as a case study. We inoculated field plots of wheat (Triticum aestivum) with two distinct Z. tritici strains. Next, we measured disease intensity as counts of fruiting bodies (pycnidia) using automated image analysis. These measurements characterized primary disease gradients, which we used to estimate effective dispersal of the pathogen population. Genotyping of reisolated pathogen strains with strain-specific PCR confirmed the conclusions drawn from phenotypic data. Consistently with controlled environment studies, we found that the characteristic scale of dispersal is tens of centimeters. We analyzed the data using a spatially explicit mathematical model that incorporates the spatial extent of the source, rather than assuming a point source, which allows for a more accurate estimation of dispersal kernels. We employed bootstrapping methods for statistical testing and adopted a two-dimensional hypotheses test based on kernel density estimation, enabling more robust inference compared with standard methods. We report the first estimates of dispersal kernels of the pathogen in field conditions. However, repeating the experiment in other environments would lead to more robust conclusions. We put forward advanced methodology that paves the way for further measurements of plant pathogen dispersal in field conditions, which can inform spatially targeted plant disease management.
\end{abstract}

Keywords: dispersal kernel, dispersal gradient, Zymoseptoria tritici, wheat, rainsplash dispersal, bootstrap, spatially explicit modeling, plant disease epidemiology, dispersal ecology

Ability to spread within host populations is a fundamental requirement for plant pathogens. Spatial spread influences the number of host plants that a pathogen can infect and governs the spatial distribution of pathogen populations. For polycyclic diseases, 
even small differences in the spread during one infection cycle can result in considerable differences in epidemic outcomes after several cycles. Understanding the mechanisms and scales of pathogen dispersal can improve our ability to predict and control plant disease epidemics.

Although the epidemiological importance of dispersal has long been recognized (Heald 1913), measurements in field conditions are rare, for numerous reasons: (i) field experiments on dispersal are difficult to design and conduct (McCartney et al. 2006); (ii) they are inherently multidisciplinary, spanning the interface between biology and physics; (iii) it is challenging to measure the primary dispersal gradient (i.e., resulting from a single round of pathogen reproduction) independently of secondary dispersal and environmental gradients (Gregory 1968); and (iv) characterization of finer-scale dispersal occurring on submeter scales can be difficult in the field. Existing literature contains many more studies characterizing airborne dispersal of plant pathogens compared with splash dispersal (McCartney et al. 2006): Fitt et al. (1987) found 305 datasets on airborne dispersal in the field, only 10 datasets on splash dispersal in controlled conditions, and no studies on splash dispersal in the field. A possible explanation for this disparity is that commercially available spore traps or disease assessments readily capture spatial scales of meters or longer, characteristic of airborne dispersal, but those are more challenging to apply over submeter scales typical for splash dispersal.

Zymoseptoria tritici (formerly Mycosphaerella graminicola) is a major fungal pathogen of wheat (Dean et al. 2012; Jørgensen et al. 2014) that causes Septoria tritici blotch (STB) disease. Z. tritici disperses by means of airborne ascospores (sexual) and splash-borne pycnidiospores (asexual). Pycnidiospores are the main driver of polycyclic epidemics during the wheat growing season. Ascospores are the main source of primary inoculum in the beginning of an epidemic and contribute to epidemic development during the season (Duvivier et al. 2013; Zhan et al. 1998, 2000). According to some studies, initial inoculum via airborne ascospores is uniform across the horizontal spatial extent of wheat fields and does not represent a limiting factor for epidemic development (Morais et al. 2016), implying that vertical dispersal from lower to higher leaf layers is more relevant epidemiologically than horizontal dispersal across the spatial extent of wheat fields. However, it is not clear how general these conclusions are; it is possible that they hold only in regions with a temperate wet climate and intense wheat production dominated by STB-susceptible wheat cultivars, where the studies have been conducted. However, most of the research on splash dispersal of $Z$. tritici has focused on vertical dispersal of spores from initial infection on basal leaves of seedlings to emerging leaf layers (Bannon and Cooke 1998; Lovell et al. 1997, 2004b; Shaw 1987; Vidal et al. 2018). In this context, the interaction between the pathogen and its host plant has been described as a "race," where the pathogen population must "climb" up to the next leaf layer before the current layer becomes senescent and resources are depleted (Robert et al. 2018).

We argue that horizontal dispersal is as important as vertical dispersal because it greatly influences the ability of a specific pathogen genotype to take over a field, and therefore, it can play a major role in the dynamics of emerging pathogen genotypes adapted to control measures such as fungicides or disease resistance genes in host plant. However, because of insufficient empirical knowledge on rain splash-driven horizontal dispersal, it has not been considered in modeling the emergence of new pathogen strains (e.g., Mikaberidze et al. 2017; Willocquet et al. 2020). Horizontal expansion of disease foci (Zadoks and van den Bosch 1994) corresponds to the growth of a genetically uniform pathogen population that is well adapted to control measures applied in a spatially uniform manner (e.g., fungicides or disease resistance genes). For this reason, spatially heterogeneous control strategies have been suggested, such as varietal mixtures in individual fields or landscape mosaics (Ben M'Barek et al. 2020; Djidjou-Demasse et al. 2017; Mikaberidze et al. 2014; Mundt and Browning 1985; Newton and Guy 2011; Newton et al. 2009; Orellana-Torrejon et al. 2021; Sapoukhina et al. 2010). The spatial scale of such strategies can be optimized based on the knowledge on spatial scales of horizontal dispersal of the pathogen. This knowledge is captured by a dispersal kernel function that describes the probability of an individual dispersal event to end up at a certain location relative to the source (Nathan et al. 2012).

Dispersal via asexual spores of $Z$. tritici and of the wheat pathogen Parastagonospora nodorum (formerly Septoria nodorum) has been studied in controlled conditions using inoculations via infected straw or spore suspension combined with artificial rain (Brennan et al. 1985; Saint-Jean et al. 2004; Vidal et al. 2017). Bannon and Cooke (1998) studied the effect of wheat-clover intercrop on dispersal from plates by artificial rain and detected a reduction of dispersed spores at $15 \mathrm{~cm}$ distance from the source. No experiment has been conducted within a host plant canopy in the field that would allow for parameterizing the dispersal kernels associated with splash dispersal.

We conducted an experiment to measure the splash dispersal of Z. tritici in field conditions. We carried out localized artificial inoculations with two $Z$. tritici strains as well as their mixture that contained equal proportions of each strain. Conducive weather conditions allowed us to measure primary dispersal gradients resulting from single dispersal events following the initial artificial inoculation. We used automated digital image analysis (Karisto et al. 2018; Stewart et al. 2016) to estimate the sizes of pathogen populations on wheat leaves based on detection of pycnidia (fungal fruiting bodies). In addition, we genotyped the reisolated strains using strain-specific primers to differentiate the inoculated pathogen populations from the background natural population. Using a spatially explicit mathematical model that considered the spatial extent of the source area (Karisto et al. $2019 b$ ), we achieved the first estimates of dispersal kernel parameters associated with splash dispersal of $Z$. tritici pycnidiospores in field conditions.

\section{MATERIALS AND METHODS}

\section{Plant materials and agronomic practices}

The experiment was performed at the Field Phenotyping Platform site, Eschikon Field Station, ETH Zurich, Switzerland (Kirchgessner et al. 2017). Experimental plots were sown with winter wheat (Triticum aestivum) cultivar Runal (breeder: Swiss Federal Research Station for Agroecology and Agriculture, FAL, Zurich, Switzerland) on 1 November 2016. Sowing density was 440 seeds $/ \mathrm{m}^{2}$, and the observed stem density on 19 June 2017 was $730 \mathrm{stems} / \mathrm{m}^{2}$. Field maintenance included herbicide Herold SC (0.6 liter/ha; Bayer) on 2 November 2016 and stem shortener Moddus (0.5 liter/ha; Syngenta) on 13 April 2017. The fungicide Input (1.25 liter/ha; spiroxamine $300 \mathrm{~g} /$ liter, prothioconazole $160 \mathrm{~g} / \mathrm{liter}$; Bayer) was applied on 13 March 2017 to suppress the natural epidemic of STB and other fungal diseases.

A similar experiment was prepared at INRAE Bioger, Thiverval-Grignon, France (coordinates: 48.840N, 1.952E). The experimental design was slightly adapted. Due to unconducive weather conditions, the inoculation did not produce measurable primary disease gradients. Therefore, no data are presented. 


\section{Experimental design}

The experimental plots were $1.125 \times 4 \mathrm{~m}$ rectangles consisting of nine 4-m-long rows with a distance of $12.5 \mathrm{~cm}$ between the rows. Plots were randomly assigned to four treatments with five replicates of each treatment, as shown in Figure 1A. The four treatments were (i) inoculation with the single strain ST99CH_1A5 (short identifier 1A5), (ii) inoculation with the single strain ST99CH_3D7 (short identifier 3D7), (iii) inoculation with both strains $1 \mathrm{~A} 5$ and 3D7 (mixed inoculation), and (iv) control (no inoculation). The two strains were collected and isolated in Switzerland in 1999 (Zhan et al. 2002; see also http://www. septoria-tritici-blotch.net/isolate-collections.html). The strains were chosen because of their capacity to infect cultivar Runal, their contrasting production of pycnidia (Stewart et al. 2018), and their ability to cross with each other (1A5, Ma1-1; 3D7, Mat1-2; Suffert et al. 2018).

In each plot, a $40-\mathrm{cm}$-wide area across the middle of the plot was inoculated. Disease levels were measured in the middle of the inoculated area $\left(\mathrm{x}_{0}=0 \mathrm{~cm}\right)$ and at eight locations outside of the inoculated area, four on each side (at distances of $x_{ \pm 1}=40 \mathrm{~cm}$,

\section{A $\leftarrow$ North-West +}
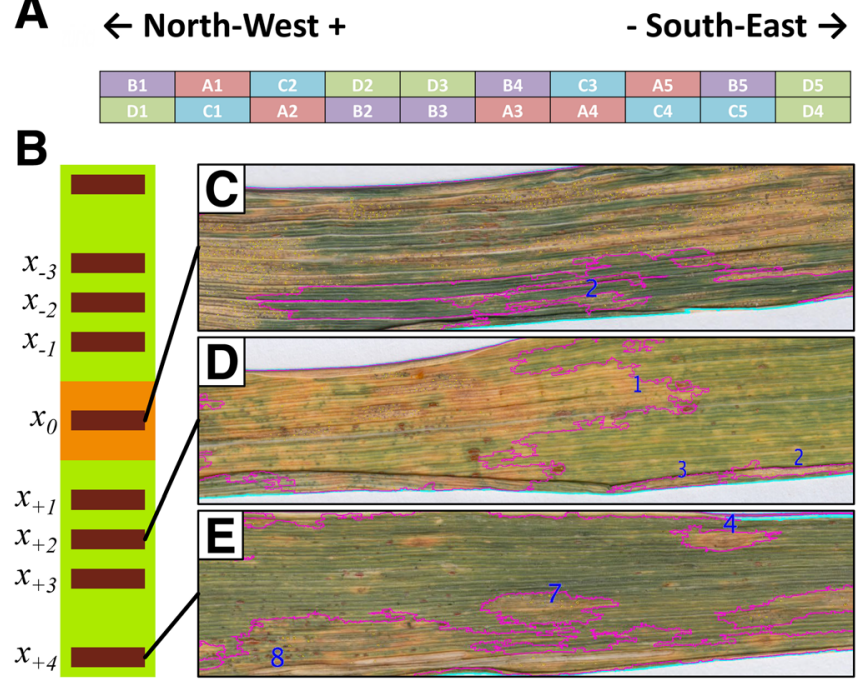

$\mathbf{F}$

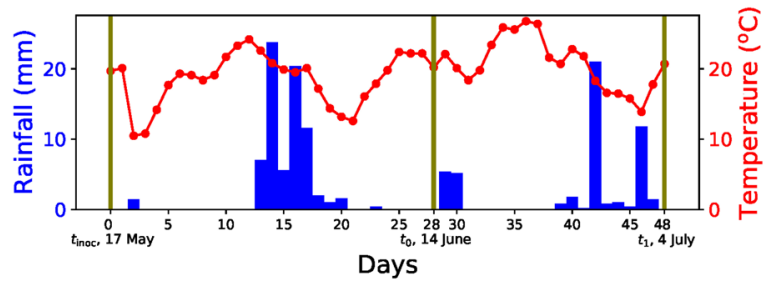

FIGURE 1

Overview of the experiment. A, Arrangement of the plots in the field (treatment letter and replicate number: $A=1 A 5, B=$ mixed inoculation, $\mathrm{C}=$ control, $\mathrm{D}=3 \mathrm{D} 7)$. $\mathrm{B}$, Design of an experimental plot; $40-\mathrm{cm}$-wide inoculation area in the middle (orange).

Distances from the middle of the inoculated area to the middle of each measurement line were $x_{0}=0 \mathrm{~cm}, x_{ \pm 1}=40 \mathrm{~cm}, x_{ \pm 2}=$ $60 \mathrm{~cm}, x_{ \pm 3}=80 \mathrm{~cm}$, and $x_{ \pm 4}=120 \mathrm{~cm}$. C, D, and $\mathbf{E}$, Overlay images illustrate the automated image analysis by showing leaves collected from measurement lines $x_{0}(C), x_{+2}(D)$, and $x_{+4}$ (E) of the treatment 3D7 at the sampling date $t_{1}$. Cyan, purple, and yellow lines mark the borders of leaves, lesions, and pycnidia, respectively. $\mathbf{F}$, Weather conditions during the experiment. Daily precipitation in the blue bars, daily mean temperature in the red line, dates of inoculation and leaf samplings are shown with vertical lines. $\mathrm{x}_{ \pm 2}=60 \mathrm{~cm}, \mathrm{x}_{ \pm 3}=80 \mathrm{~cm}$, and $\mathrm{x}_{ \pm 4}=120 \mathrm{~cm}$ from the center of the inoculated area; see Fig. 1B). The areas in which we conducted measurements ("measurement lines," shown as dark rectangles in Fig. 1B) were $10 \mathrm{~cm}$ wide and $87.5 \mathrm{~cm}$ long across the plot, excluding the $12.5-\mathrm{cm}$ borders at each edge of the plot to reduce edge effects. Disease assessments were conducted uniformly over the area of each measurement line.

\section{Z. tritici inoculation}

Inoculum was prepared by growing the fungus for 7 days in yeast-sucrose broth (https://dx.doi.org/10.17504/protocols.io. mctc $2 w n)$. The liquid culture was then filtered and the blastospores were pelleted in centrifuge and resuspended in sterile water. The washed spore suspension was diluted with water to achieve the concentration of $10^{6}$ spores $/ \mathrm{ml}$. For mixed inoculation, the spore suspension was obtained by mixing the same volume of each single-strain suspension so that the final spore concentration was $10^{6}$ spores $/ \mathrm{ml}$ and each strain was present with the concentration of $5 \times 10^{5}$ spores $/ \mathrm{ml}$. Finally, we added $0.1 \%$ (v/v) of Tween 20 and kept the inoculum suspension on ice until spraying on the same days.

Inoculation was performed by spraying $300 \mathrm{ml}$ of spore suspension onto the inoculation site of each plot using a hand-pump pressurized sprayer. The plots were inoculated during the late afternoon to avoid direct sunlight. All treatments were inoculated with the same sprayer, which was rinsed with water and with $70 \%$ ethanol to clean all parts before inoculating each treatment. The entire canopy within the inoculation area was inoculated until runoff. During the spraying, the $40-\mathrm{cm}$-wide inoculation area was bordered with plastic sheets to avoid spillover of the inoculum outside the area. After spraying, the border sheets were folded over the canopy to enclose the plants in a plastic tent to maintain high humidity overnight. The tents were removed early the next morning to avoid overheating. The inoculation was repeated the next evening. Photographs documenting the inoculation process are shown in Supplementary File S1 (Appendix B).

We first inoculated the plants on 5 to 6 April, when the F-3 layer (the third leaf layer below the flag leaf) had mostly emerged (approximate growth stage, GS 22; Zadoks et al. 1974), but this inoculation did not cause sufficient disease (see below). For this reason, we conducted a second inoculation on 17 and 18 May, when flag leaves had already emerged (GS 39 to 41). The second inoculation resulted in strong, measurable disease gradients.

We considered the first inoculation as failed for the purpose of the experiment based on three lines of evidence. First, when we assessed the inoculation success on 24 April and 3 May, infection was extremely limited. On 3 May, we observed low levels of disease in the F-3 leaf layer when the plants were in the beginning of stem elongation (F-1 emerging, GS 35). Average STB incidence in the F-3 layer in the inoculated areas was 6.1, 4.9, 2.9 , and $0 \%$ for strains $1 \mathrm{~A} 5,3 \mathrm{D} 7$, mixed inoculation, and control, respectively. In all the leaf layers above F-3, we observed disease incidence close to zero (one lesion across 655 tillers). Second, the top three leaf layers consistently remained healthy until 8 June. Only on 14 June (28 days after inoculation, DAI) did we observe for the first time substantial numbers of sporulating lesions on the top three leaf layers within the inoculated areas. A rapid onset of symptoms must have occurred between 8 June (when we observed only a few tiny lesions, mostly without pycnidia) and 14 June. This onset of symptoms matched with the date of the second inoculation, considering the estimated latent period. Third, the most plausible reason the first inoculation did not cause substantial disease was that April 2017 was unusually cold, including 3 days (18 to 20 April) with minimum temperatures below zero, 
which are known to hinder the development of disease. To conclude, it is extremely unlikely that the first inoculation had any measurable effect on the observed disease gradients.

\section{Assessment of disease gradients}

The disease assessment combined incidence and severity measurements. It was performed at two time points. At $\mathrm{t}_{0}$, on $14 \mathrm{June}$ 2017 (GS 70), only the inoculation areas were assessed carefully to evaluate the success of inoculation across the measurement line $\mathrm{x}_{0}$. Flag leaves outside the inoculation area were inspected visually and confirmed to be healthy. At $\mathrm{t}_{1}$ on 4 July 2017 (GS $85)$, all measurement lines $\left(\mathrm{x}_{0}, \mathrm{x}_{ \pm 1}, \mathrm{x}_{ \pm 2}, \mathrm{x}_{ \pm 3}\right.$, and $\left.\mathrm{x}_{ \pm 4}\right)$ were assessed in all plots corresponding to inoculated treatments (1A5, 3D7, and mixed inoculation), whereas only one randomly chosen measurement line was assessed in each of the control plots.

At $t_{0}$, STB incidence was measured at the leaf scale in the following manner. Thirty to 40 stems were inspected on each measurement line. The highest diseased leaf layer was recorded for each stem. The leaves lower than that were assumed to be diseased, as STB is usually more prevalent in the lower leaf layers (Lovell et al. 2004a). Additionally, naturally senescent leaf layers at the bottom of the canopy were recorded. In this way, incidence was estimated for all nonsenescent leaf layers. After estimating the incidence, eight infected leaves were collected from up to two consecutive leaf layers that had incidence higher than $20 \%$ (to avoid removing too much of the inoculum). The collected leaves were then mounted on paper sheets and scanned with 1,200 dpi resolution. The resulting images were analyzed using an automated image analysis method to measure two aspects of conditional STB intensity that represent the host damage and the pathogen reproduction, as described by Karisto et al. (2018). Host damage was measured as the percentage of leaf area covered by lesions, and pathogen reproduction was measured as the number of pycnidia per leaf. The sampled leaf layers at $t_{0}$ were the flag leaf layer $(\mathrm{F})$ and the layer immediately below the flag leaf $(\mathrm{F}-1)$.

At $t_{1}$, the wheat leaves were already mostly chlorotic and hence the incidence measurement was not possible in the field. Instead, we collected at random about 24 leaves from each measurement line. The leaves were taken into the lab and inspected for the presence of pycnidia. Incidence was recorded based on the presence of pycnidia, and the leaves with pycnidia were scanned as described above to quantify the conditional STB intensity. Due to vast chlorosis, the measurement of host damage was considered unreliable, and only pathogen reproduction was used in the subsequent analysis. Thus, we measured the conditional disease intensity using only the numbers of pycnidia per leaf.

We estimated the number of asexual reproduction and dispersal events between $t_{0}$ and $t_{1}$ using the following arguments. First, based on the data from Shaw (1990; as revisited in Figure A1 of Karisto et al. 2018), the latent period after inoculation was estimated to be longer than 20 days (average daily temperature during first 19 days was $19^{\circ} \mathrm{C}$ ). Thus, there was likely no spread from the inoculation areas during the rainy period 13 to $17 \mathrm{DAI}$ (Fig. 1F). This was further confirmed by a visual assessment on 8 June (22 DAI), when we observed only a few tiny lesions and mostly no pycnidia on plants inside the inoculation areas, concluding that substantial spread had not yet occurred. Second, at $\mathrm{t}_{0}$ (14 June, $28 \mathrm{DAI}$ ) we observed substantial disease (Fig. $2 \mathrm{~A})$ in the inoculation areas. There was no rain during the week preceding $\mathrm{t}_{0}$, two strong showers during the night, about $36 \mathrm{~h}$ after $\mathrm{t}_{0}$, and no more rain during the following week. We concluded that there was most likely only one asexual spread event right after $t_{0}$, which caused the disease gradients that we observed at $\mathrm{t}_{1}$ (4 July, 48 DAI) outside of the inoculation areas.

We concluded that the second inoculation was successful: It caused high disease levels observed in the inoculation areas at $t_{0}$, after a latent period of 3 to 4 weeks. After 3 more weeks, at $t_{1}$, there were clearly visible symptoms outside of the inoculation areas. The observed symptoms at $t_{1}$ can be entirely attributed to the rain event on 17 June (shortly after $t_{0}$ ) and consequent asexual spread of the pathogen.

\section{Genotyping}

To confirm that the observed disease gradients were due to the experimental treatments, we reisolated $153 \mathrm{Z}$. tritici individuals from individual pycnidia on the collected leaves after scanning. The isolations were performed from leaves collected in the measurement lines $\mathrm{x}_{ \pm 1}$ and $\mathrm{x}_{ \pm 3}$. To detect the inoculated strains, we designed and used strain-specific PCR primers (see details in the Supplementary File S1, Appendix A). As a result of the genotyping, the isolates were identified as either 1A5 or 3D7 if the two primer pairs specific to one of these strains yielded amplification whereas the primer pairs specific to the other strain did not.

\section{Statistical analysis}

Fitting disease gradients. The disease intensity (numbers of pycnidia per leaf) at $t_{1}$ in a given measurement line is a result of dispersal of asexual spores from the inoculation area and successful infections within the measurement line. Assuming spatially uniform success of infections in all plots, the observed disease gradient reflects the dispersal gradient of spores and thus provides the effective dispersal gradient of the pathogen population. Following the spatially explicit method for parameterizing a dispersal kernel (Karisto et al. 2019b), the dispersal process can be described mathematically using two area integrals: one over the source area and the other over the destination area. We used the exponential dispersal kernel:

$$
\kappa(r)=\frac{e^{-\frac{r}{\alpha}}}{2 \pi \alpha^{2}}
$$

that fits the experimental data well for splash dispersal (Fitt et al. 1987; Saint-Jean et al. 2004). Here, $r$ is the distance from the source to the destination of dispersal and $\alpha$ is the scale parameter of the dispersal kernel that determines the characteristic spatial scale of dispersal (below we call it the "dispersal parameter").

As the dispersal kernel characterizes a point-to-point process, we consider the area of the inoculated source $(S)$ and area of the destination $(D)$ by integrating over those areas, which gives us the total disease intensity transmitted from the source to a measurement line. The average intensity within a measurement line is calculated by dividing the total intensity by the area of the measurement line $\left(A_{D}\right)$. Hence, the disease intensity at $t_{1}$ in a measurement line at a distance $x^{*}$ from the inoculation area is given by

$$
I_{t_{1}}\left(x^{*}\right)=\frac{I_{0} \beta}{\mathrm{A}_{\mathrm{D}}} \iint_{D\left(x^{*}\right)} \iint_{S} \frac{e^{-\frac{\sqrt{\left(x_{d}-x_{s}\right)^{2}+\left(y_{d}-y_{s}\right)^{2}}}{\alpha}}}{2 \pi \alpha^{2}} d A_{S} d A_{D}
$$

This general approach can consider arbitrary shapes of the two areas of integration $S$ and $D$. In the specific case of our experimental design, the two areas correspond to the following rectangles: $\mathrm{S}=\left\{\left(x_{s}, y_{s}\right)\right\}=\left[0,1_{\mathrm{S}}\right] \times[0, \mathrm{w}]$ is the source area that corresponds to the inoculated area (the orange rectangle in Fig. 1B), and $\mathrm{D}=\left\{\left(x_{d}, y_{d}\right)\right\}=\left[\mathrm{x}^{*}-\mathrm{w}_{\mathrm{d}} / 2, \mathrm{x}^{*}+\mathrm{w}_{\mathrm{d}} / 2\right] \times[\mathrm{b}, \mathrm{w}-\mathrm{b}]$ is 
the destination area that corresponds to a measurement line (the dark rectangles in Fig. 1B). $\mathrm{I}_{0}$ is the STB intensity (numbers of pycnidia per leaf) in the inoculation area at $\mathrm{t}_{0} ; \beta$ is the transmission rate (unitless) describing the number of new pycnidia produced in the measured leaf layer per unit of measured intensity in the source leaves; $1_{\mathrm{s}}=40 \mathrm{~cm}$ is the extent of the inoculated area along the $x$ axis (i.e., along the length of the plot); $w=112.5$ $\mathrm{cm}$ is the plot width; $\mathrm{w}_{\mathrm{d}}=10 \mathrm{~cm}$ is the width of measurement lines; and $b=12.5 \mathrm{~cm}$ is the width of the border excluded from the measurement lines. Hence, the integration over a measurement line divided by the area of the line, $A_{D}=w_{d}(w-2 b)$, gives the average disease intensity across the measurement line, reflecting spatially uniform leaf sampling.

Note that the $10-\mathrm{cm}$ width of the measurement lines was practically the smallest possible width that could be achieved in the field measurements because the foliage of even a single straw spans more than $10 \mathrm{~cm}$, limiting the spatial resolution of our measurements. For this reason, we simplified the model by neglecting the width of the measurement lines and assigning the disease intensity values of each measurement line to the middle of the line. With this simplification, equation 2 reads

$I_{t_{1}}\left(x^{*}\right)=\frac{I_{0} \beta}{w-2 b} \int_{b}^{w-b} \int_{0}^{l_{s}} \int_{0}^{w} \frac{e^{-\frac{\sqrt{\left(x^{*}-x_{s}\right)^{2}+\left(y_{d}-y_{s}\right)^{2}}}{\alpha}}}{2 \pi \alpha^{2}} d x_{s} d y_{s} d y_{d}$

Dispersal is often modeled as a one-dimensional process (Fitt et al. 1987; Madden et al. 2007), which neglects the spatial extent of the source area, assuming it to be a point. This approximation usually leads to inaccurate estimates of dispersal kernels (Karisto et al. 2019b). For this reason, we used the spatially explicit model equation 3, which allows for more accurate estimation of dispersal kernels. However, to enable comparisons between our outcomes and the earlier estimates in controlled conditions, and to highlight the distorting effect of this "standard" approximation, we constructed the one-dimensional function describing the dispersal process
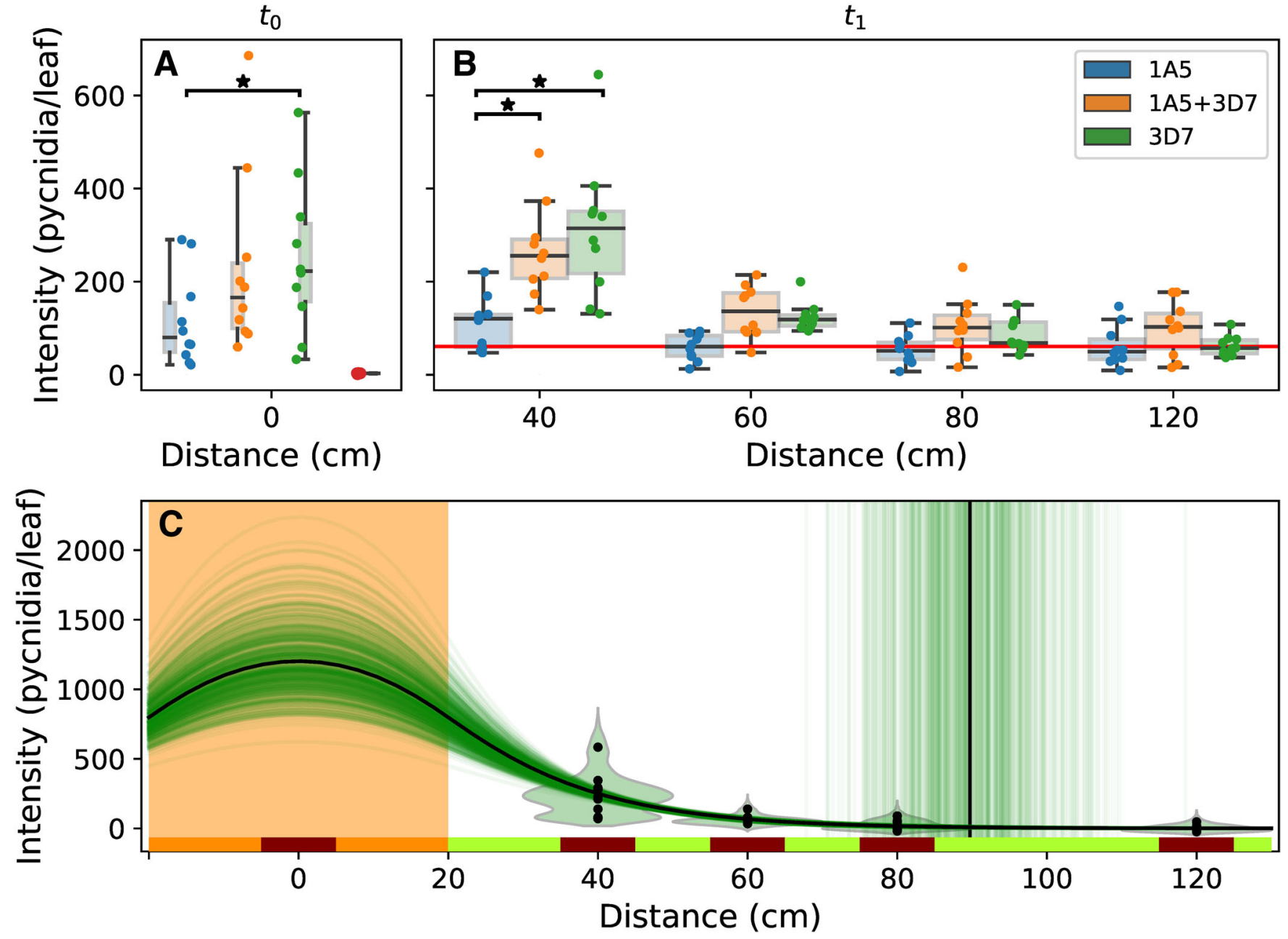

FIGURE 2

Disease intensity and dispersal gradients. In all panels, dots represent means over individual replicate plots. In panels $A$ and $B$, we present outcomes for 1A5 (blue), mixed inoculation 1A5+3D7 (orange), 3D7 (green), and noninoculated control (red) treatments. Black horizontal bars with asterisks show the significant pairwise differences between treatments at $t_{0}$ and at $t_{1}$. A, Disease intensity in the inoculation area at $\mathrm{t}_{0}$, measurements for leaf layers $\mathrm{F}$ and $\mathrm{F}-1$ for the three treatments, $\mathrm{F}-2$ for control (as there was no disease at higher leaf layers). B, Gradients of disease intensity in leaf layer $F$ at $t_{1}$ after the spread event for the three inoculation treatments. Red horizontal line shows the mean disease intensity in F layer over all control plots. C, Disease gradients of strain 3D7 are fitted using equation 3. Black curve shows the best fit, vertical black line shows 99 th percentile of the dispersal distance $\left(d_{99}\right)$. Green violin plots show the distributions of 500 bootstrap replicates, green curves represent fits to each of these bootstrap replicates, and green vertical lines show the corresponding $d_{99}$ values. 
that follows an exponential kernel:

$$
I_{t_{1}}\left(x^{*}\right)=I_{0} \beta \int_{0}^{l_{s}} \frac{e^{-\frac{\left|x^{*}-x_{s}\right|}{\alpha}}}{2 \alpha} d x_{s}
$$

In equation 4 , the integral takes sum over the inoculated area along the length of the plot. We conducted the integration in equation 4 and obtained a simpler expression:

$$
I_{t_{1}}\left(x^{*}\right)=\frac{I_{0} \beta}{2}\left(e^{\frac{l_{s}}{\alpha}}-1\right) e^{-\frac{x^{*}}{\alpha}}
$$

where $x^{*}$ takes the values outside of the inoculation area.

\section{Estimation of dispersal and transmission parameters}

The data used in the analysis were obtained in the following way. First, we conducted STB incidence measurements and acquired conditional STB intensity measurements from the digital image analysis. Second, the STB intensity values in each measurement line were multiplied by the corresponding STB incidence to obtain the full or "unconditional" intensity values, hereafter called "intensity" for the sake of simplicity. Third, we calculated the average full intensity in each measurement line to obtain five data points (from five replicates) for each treatment (at distances $\mathrm{x}_{0}, \mathrm{x}_{ \pm 1}, \mathrm{x}_{ \pm 2}, \mathrm{x}_{ \pm 3}$, and $\left.\mathrm{x}_{ \pm 4}\right)$. These average values over each measurement line were used for fitting the model functions. The dispersal gradient functions (equations 2 and 3 ) were fitted to the data to estimate $\alpha$ and $\mathrm{I}_{0} \beta$.

To compare the treatments and the two directions, we used the bootstrapping approach. We resampled the collected samples with replacement to create a large set of bootstrap samples. Variation in the bootstrap samples reflects the variation that we would expect to observe if the actual experiment was repeated several times (see, e.g., Davison and Hinkley 1997). Bootstrapping allowed us to model explicitly the variation related to the incidence counts and the variation related to the leaf collection, independently of each other. This approach also allowed us to assess uncertainties in the parameter estimates without making any assumptions about the distributions of the data or the parameter values.

We created 100,000 bootstrap samples for each measurement line in each replicate in the following manner. First, we simulated the incidence counts on the measurement lines to create a distribution of incidence values. We assumed a population of 82 stems within each measurement line (based on the observed stem density) and simulated a random leaf sampling 100,000 times with all possible incidence values. We recorded the "real" incidence value each time the simulation produced the observed value. That created a distribution of "real" incidence values corresponding to our observation. Second, we sampled with replacement the analyzed leaves to generate 100,000 new samples with the original sample size. Third, the mean disease intensity of each bootstrap leaf set was multiplied by an incidence value drawn from the corresponding incidence distribution to obtain the mean intensity for each measurement line. Finally, we grouped these unconditional means over measurement lines into sets representing the five replicates. As a result, we obtained 100,000 bootstrap replicates of the entire experiment.

The one-dimensional disease gradient in equation 5 was fitted to each of the 100,000 bootstrap replicates. The disease gradient function in equation 3 was fitted to a subset of 10,000 bootstrap replicate datasets for computational reasons. As a result, we obtained a large number of bootstrap estimates of parameters $\alpha$ and $\beta I_{0}$ for each treatment and direction. These estimates were used to conduct statistical tests.

\section{Statistical tests}

Parameter differences were tested using a simple bootstrap hypothesis test (Davison and Hinkley 1997, p. 162), where the observed difference between parameter values in different conditions is compared to a distribution of differences between those conditions in the bootstrap samples. If only a few bootstrap samples give a more extreme difference than the observed one, then the observed difference is considered significant. Significance level ( $P$ value) of the observed difference is calculated by dividing the number of cases where the difference in the test statistic $s_{i}$ is greater than or equal to the observed difference $\mathrm{s}_{\text {obs }}$ (including the observed case) by the total the number of bootstrap replicates $(R)$ plus the observed case:

$$
P=\frac{1+\#\left\{s_{i} \geq s_{o b s}\right\}}{1+R}
$$

Additionally, we tested differences between conditions in the parameters $\alpha$ and $\beta \mathrm{I}_{0}$ simultaneously using a two-dimensional hypothesis test based on the joint distribution of differences in $\alpha$ and $\beta \mathrm{I}_{0}$ (the "equidensity" test, analogous to Johansson et al. 2014). A kernel density estimate of the joint distribution was obtained to define the degree of "extremity" of a point in the twodimensional parameter space. The point reflecting the observed difference was compared to the joint distribution of differences between bootstrap replicates. The observed difference was considered significantly different from zero if it was located in a sufficiently sparse area, such that less than 5\% of the bootstrap estimates were located in regions with equal or lower density, analogous to being in the thin tail of a one-dimensional distribution.

We present $95 \%$ confidence intervals for the parameters derived from the distribution of bootstrap results (i.e., the limits of the 2.5th and 97.5th percentile of the distribution). Additionally, differences in disease levels between treatments 1A5, 3D7, and mixed inoculation were tested at $t_{0}, x_{0}$ and $t_{1}, x_{ \pm 1}$ with the Kruskal-Wallis test and the pairwise Dunn's post hoc comparison with Bonferroni correction.

\section{Statistics implementation}

All data analysis was implemented in Python (versions 3.5.2 and 3.6.0), and the code is provided together with the data. Fitting was performed using the 'Model.fit' function of the $1 \mathrm{~m}$ fit package (v. 0.9.10; Newville et al. 2014), with the methods 'brute' for initial guess, 'leastsq' for actual fitting, and occasionally 'tnc' (truncated Newton) when 'leastsq' failed. Numerical integrations were implemented with 'quad' and 'dblquad' functions in the SciPy package (v. 1.0.1; Virtanen et al. 2020). Fitting of the two-dimensional functions (equations 2 and 3) was performed using the high-performance computing cluster Euler of ETH Zurich. The Kruskal-Wallis test was conducted with the 'Kruskal' function in the SciPy package and Dunn's test with the 'posthoc_dunn' function in the scikit-posthocs package (v. 0.3.8; Terpilowski 2018).

\section{RESULTS}

The second inoculations with strains $1 \mathrm{~A} 5$ and 3D7, and their mixture, were successful: At $t_{0}$, we observed increased disease levels in the inoculation areas of all three treatments compared with controls (Fig. 2A). At time point $t_{1}$, there was a gradient of disease intensity from higher levels at $\mathrm{x}_{ \pm 1}$ to lower levels at $\mathrm{x}_{ \pm 4}$ (Fig. 2B). Genotyping the reisolated strains confirmed the successful spread (Supplementary File S1, Appendix A). In to- 
tal, 4,190 plants were inspected in the course of incidence measurements, 2,527 leaves were collected and analyzed using the digital image analysis, and 153 isolates were genotyped. The entire dataset including raw data, bootstrap replicates, best fitting parameter estimates, and weather data is available in Dryad data repository (https://doi.org/10.5061/dryad.kkwh70s5r).

\section{Estimates of pathogen dispersal parameters}

Fitting equation 3 to the observed disease gradients allowed us to estimate parameters $\alpha$ (dispersal parameter) and $\beta \mathrm{I}_{0}$ (transmission rate $\times$ initial intensity at the source). For $1 \mathrm{~A} 5$ treatment, the estimates of $\alpha$ were very low and estimates of $\beta \mathrm{I}_{0}$ were very high compared with 3D7 and mixed inoculation (Table 1). The results are biologically unrealistic and were likely due to an insufficient disease intensity within the inoculation area and consequently a shallow gradient outside the inoculation area (Fig. 2A and B). Less successful spread of 1A5 than of 3D7 was confirmed by comparing disease intensities between the treatments at $\mathrm{t}_{0}, \mathrm{x}_{0}$ and at $t_{1}, x_{ \pm 1}$. At $t_{0}, x_{0}$, the disease intensity was significantly lower for 1A5 than for 3D7 (Kruskal-Wallis test $\mathrm{H}=10.66$, $P=0.005$; pairwise Dunn's test $P=0.004$ ). Furthermore, at $\mathrm{t}_{1}$, $\mathrm{X}_{ \pm 1}$, the intensity in treatment $1 \mathrm{~A} 5$ was lower than the intensity in both the 3D7 and the mixed inoculation treatments (KruskalWallis $\mathrm{H}=117.1, P=3.4 \times 10^{-26}$; Dunn's test 1 A5 versus 3 D7 $P=6.0 \times 10^{-24}$; Dunn's test 1 A5 versus mixed inoculation $P=2.6 \times 10^{-18}$ ); at other measurement distances, the KruskalWallis test detected no differences. Due to the differences in 1A5 treatment compared with others, the next steps of analysis are presented only for 3D7 and mixed inoculation treatments.

Comparison of the best-fitting parameters (Table 1) between the "positive" (northwest) and "negative" (southeast) directions revealed no significant difference in treatment 3D7 (equidensity $P$ value: $P_{2 \mathrm{D}}=0.21$, one-dimensional hypothesis test equation 6 for parameter $\alpha: P_{\alpha}=0.17$, parameter $\beta \mathrm{I}_{0}: P_{\beta \mathrm{I}_{0}}=0.13$, Fig. 3A), nor in the mixed inoculation treatment $\left(P_{2 \mathrm{D}}=0.74\right.$, $\left.P_{\alpha}=0.60, P_{\mathrm{BI}_{0}}=0.95\right)$. This similarity between directions suggests isotropic dispersal during the experiment.

Considering this isotropy, we estimated the parameters using the dataset that combined the two directions. We established a significant difference between 3D7 and mixed inoculation treatments $\left(P_{2 \mathrm{D}}=0.014, P_{\alpha}=0.020, P_{\beta \mathrm{I}_{0}}=0.018\right.$, Fig. 3B $)$. The dispersal parameter $\alpha$ was higher in mixed inoculation, whereas $\beta \mathrm{I}_{0}$ was higher in the $3 \mathrm{D} 7$ treatment (Table 1). We thus highlight that following the mixed inoculation, the pathogen had dispersed further but caused lower disease transmission compared with 3D7 treatment.

TABLE 1

The best-fitting parameters from equation $3^{\mathrm{z}}$

\begin{tabular}{llcc}
\hline Treatment & Direction & $\alpha(95 \% \mathrm{CI})(\mathrm{cm})$ & $\beta \mathrm{I}_{0}(95 \% \mathrm{CI})(\mathrm{pycnidia} / \mathrm{leaf})$ \\
\hline 1A5 & & & \\
& Positive & 2.5 & 99,992 \\
& Negative & 4.9 & 3,518 \\
& Combined & 2.6 & 99,975
\end{tabular}

3D7

$\begin{array}{ccc}\text { Positive } & 15.3(12.3-19.7) & 1,559(1,147-2,112) \\ \text { Negative } & 12.0(9.3-15.5) & 2,387(1,613-3,638) \\ \text { Combined } & \mathbf{1 3 . 5}(\mathbf{1 1 . 5}-\mathbf{1 6 . 1}) & \mathbf{1 , 9 1 5}(\mathbf{1 , 4 7 5}-\mathbf{2 , 4 3 0}) \\ \text { Mixed inoculation } & & \\ \text { Positive } & 23.1(15.9-37.5) & 1,271(1,052-1,618) \\ \text { Negative } & 20.0(14.8-28.4) & 1,281(1,049-1,586) \\ \text { Combined } & \mathbf{2 1 . 4}(\mathbf{1 6 . 7 - 2 8 . 6}) & \mathbf{1 , 2 7 1}(\mathbf{1 , 0 9 5}-\mathbf{1 , 4 6 8})\end{array}$

\footnotetext{
${ }^{\mathrm{z}}$ Confidence intervals (CIs) derived from bootstrapping, except for the implausible results of strain 1A5. Values of $\beta I_{0}$ were limited below 100,000 in the fitting procedure.
}

\section{Estimates of disease transmission rates}

In addition to estimating the pathogen dispersal parameter, we estimated the disease transmission rate. The fitting yielded estimates of $\beta \mathrm{I}_{0}$, from which we extracted the transmission rate $\beta$ by dividing $\beta \mathrm{I}_{0}$ by estimates of $\mathrm{I}_{0}$. We calculated $\mathrm{I}_{0}=249$ pycnidia/leaf for 3D7 and $\mathrm{I}_{0}=227$ pycnidia/leaf for mixed inoculation, measured as the mean intensity in flag leaves. Based on these numbers, we calculated $\beta=7.7$ (unitless) for 3D7 and $\beta=$ 5.6 for the mixed inoculation treatment.

\section{Genotyping as a confirmatory approach}

Genotyping of the 153 reisolated strains (Supplementary File S1, Appendix A) supported the conclusions drawn from the phenotypic data. (i) The inoculated pathogen strains had spread out from the inoculated area: We detected them in the measurement
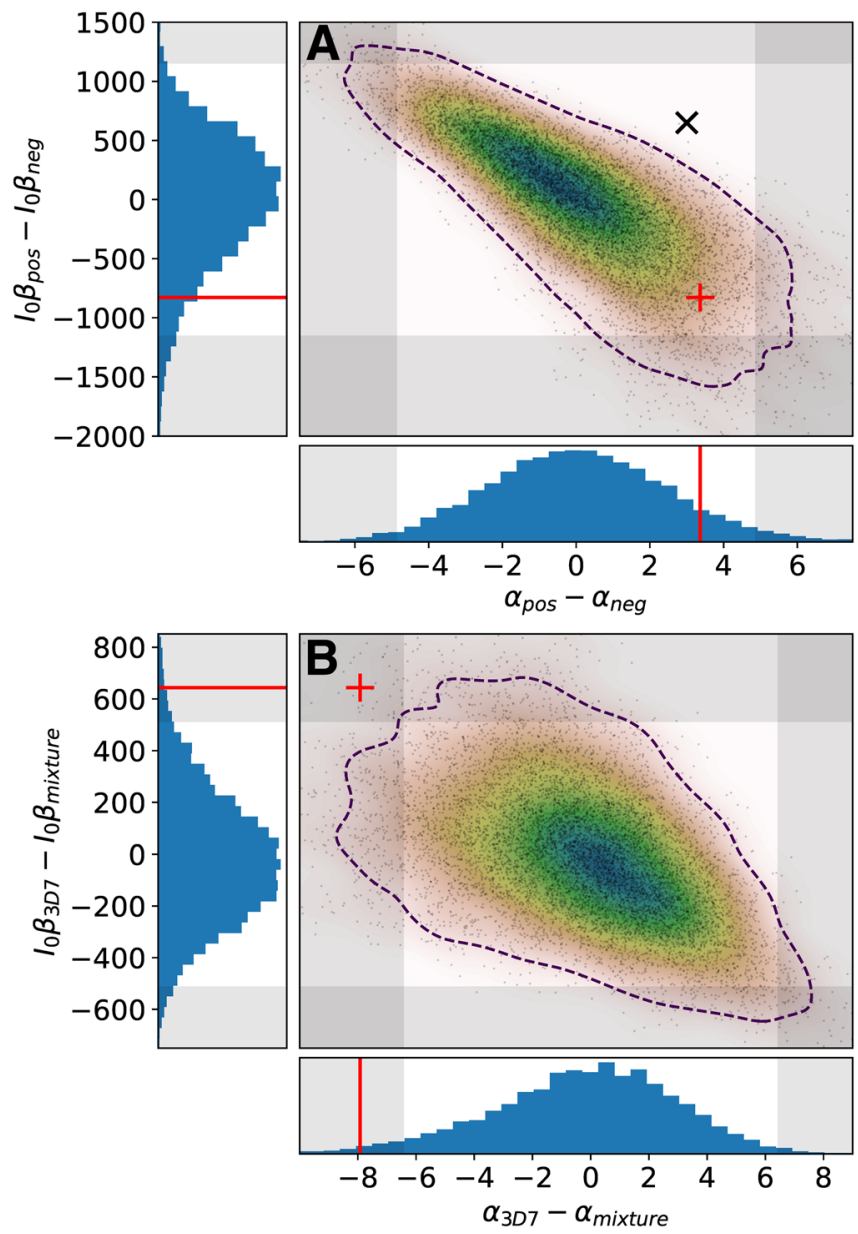

FIGURE 3

Visualization of the one- and two-dimensional bootstrap tests.

A, Comparison between two directions of dispersal of the strain 3D7. B, Comparison between 3D7 and mixed inoculation treatments. Histograms show single-parameter distributions while heat maps and black dots show joint distributions $(10,000$ bootstrap replicates). Observations (red line; red cross) in the $5 \%$ extreme of the distribution (shaded area; outside of the dashed line) are considered significant. The differences are significant between treatments $(B)$ but not between directions (A). Black cross in panel $A$ shows a hypothetical observation where the difference would be deemed nonsignificant for each parameter separately (in nonshaded area), but the joint test reveals a significant difference (outside of the dashed line). 
lines, both after inoculations with a single strain ( 9 isolates out of 19 were identified as strain $1 \mathrm{~A} 5$ at $\mathrm{x}_{ \pm 1}$ for $1 \mathrm{~A} 5$ treatment, and 45 out of 55 as 3D7 for 3D7 treatment) and after the mixed inoculation (two 1A5 isolates and 37 3D7 isolates out of the total 49 isolates sampled at $\mathrm{x}_{ \pm 1}$ versus one $1 \mathrm{~A} 5$ isolate and eight $3 \mathrm{D} 7$ isolates out of total 30 isolates sampled at $\mathrm{x}_{ \pm 3}$ ). (ii) The proportion of identified 1A5 and 3D7 isolates was lower farther away from the inoculation area in the mixed inoculation treatment. We can thus conclude that the strain 1A5 spread less successfully than the strain 3D7: The proportion of the 3D7 isolates in the 3D7 treatment was higher than the proportion of $1 \mathrm{~A} 5$ isolates in the 1 A5 treatment, and the same effect was observed in the mixed inoculation treatment.

\section{How good are the simplifications?}

We compared the three different models (equations 2, 3, 4) with regard to (i) the accuracy of the estimates and (ii) the computational time.

First, the parameter estimates are virtually the same when using the two two-dimensional models (line destination versus explicit destination area), which justifies our use of the slightly simplified model in equation 3 . In contrast, the one-dimensional model resulted in substantially higher estimates of $\alpha$ (Table 2).

Additionally, the relationship between the population spread and dispersal parameter $\alpha$ is different between one- and twodimensional models. This difference becomes clear, for example, when dispersal is described based on "mean dispersal distance," which is $\alpha$ for a one-dimensional exponential kernel but $2 \alpha$ for a two-dimensional kernel. For the strain 3D7, the mean dispersal distances in one- and two-dimensional models would be $15 \mathrm{~cm}$ and $27 \mathrm{~cm}$, respectively. Clearly, a one-dimensional model of dispersal should not be used for deriving dispersal distances.

Second, regarding the computational time, the onedimensional model (equation 4) was fitted to 100,000 bootstrap datasets on a standard PC in a few hours, whereas fitting the two-dimensional model (equation 3 ) required a few days of computational time for only 10,000 replicates. When using the most complete model (equation 2), it took more than $12 \mathrm{~h}$ on a $\mathrm{PC}$ to obtain the estimates for only one replicate (the observed data). We conclude that considering the spatial extent of the source by means of two-dimensional modeling pays off in the increased accuracy of the parameter estimates, even though the computational demand increases.

\section{DISCUSSION}

\section{Take-home message}

We measured primary, horizontal disease gradients produced by rain-splash dispersal and subsequent transmission via asexual spores of $Z$. tritici in wheat canopy. We achieved the first estimates of the dispersal kernel of the pathogen in field conditions.
We put together an advanced methodological toolbox, but further measurements in other environments are required for more robust conclusions. Consistently with studies in controlled conditions, we report that the characteristic spatial scale of dispersal is tens of centimeters. Our estimation of the dispersal kernel can be used to provide biologically plausible parameter ranges for epidemiological models that describe spatial-temporal disease dynamics within wheat fields.

\section{Measurement of the pathogen population}

The use of digital image analysis allowed us to measure pycnidia numbers within individual leaves. This measure represents an important aspect of STB disease intensity (Karisto et al. 2018; Stewart et al. 2016): It reflects the population size of the pathogen resulting from the previous infection cycle and characterizes the transmission potential of the pathogen in following infectious cycles. For these reasons, this measure is a proxy for pathogen reproduction.

A more conventional measure of disease intensity, the proportion of leaf area covered by lesions (PLACL), is associated with yield loss, but pycnidia numbers are more powerful than PLACL for predicting the STB epidemic development (Karisto et al. 2018). This is because PLACL may be affected by other physiological and developmental processes in plants, as well as environmental factors. Thus, characterization of pathogen reproduction is more relevant for describing epidemic dynamics.

\section{Characterization of effective dispersal}

The dispersal kernels estimated here correspond to effective dispersal of the pathogen population, not to dispersal of spores. A difference between those may arise from density-dependent postdispersal mortality (Klein et al. 2013; Nathan et al. 2012). At high spore densities, which can occur close to the source, leaves may become saturated, thereby decreasing the infection efficiency of the spores (Karisto et al. 2019a). Alternatively, dispersal of spores could be measured with spore traps placed within the canopy. However, that would leave unclear how many spores attach to healthy plants and how many of them are successful in causing lesions and pycnidia. Using healthy plants as spore traps leads to measurement of the combination of dispersal and infection processes that is more epidemiologically relevant.

\section{Estimation of dispersal and transmission parameters}

Dispersal parameter estimates $\alpha_{3 \mathrm{D} 7}=13.5 \mathrm{~cm}$ and $\alpha_{\mathrm{mix}}=$ $21.4 \mathrm{~cm}$ correspond to half distances $22.7 \mathrm{~cm}$ and $35.9 \mathrm{~cm}$ for $3 \mathrm{D} 7$ and mixed inoculation treatments, respectively (half-distance $\approx$ $1.7 \alpha$ in 2D; Karisto et al. 2019b). Fitt et al. (1987) report a notably shorter range of 6 to $16 \mathrm{~cm}$ for half-distances of dispersal of splash-borne fungal spores reviewed in the literature, including estimates for Z. tritici from Brennan et al. (1985), but they used

TABLE 2

Comparison of the parameter estimates between different functions ${ }^{2}$

\begin{tabular}{lcccc}
\hline & \multicolumn{2}{c}{ Mixed inoculation treatment B } & \multicolumn{2}{c}{$3 \mathrm{D}$ treatment } \\
\cline { 2 - 4 } Equation & $\alpha(\mathrm{cm})$ & $\beta \mathrm{I}_{0}$ (pycnidia/leaf) & & $\alpha(\mathrm{cm})$ \\
\hline Equation 2, 2D & 21.4 & 1,265 & 13.5 \\
Equation 3, 2D & 21.4 & 1,271 & 1,889 \\
Equation 4, 1D & 24.6 & 1,131 & 1,915 & 13.5 \\
\hline
\end{tabular}

${ }^{\mathrm{z}}$ Estimates with combined data of the two directions. "2D" and "1D" stand for two- and one-dimensional models, respectively. 
a one-dimensional model of dispersal. Our one-dimensional estimates (Table 2) correspond to half-distances of 10.5 and 17.0 $\mathrm{cm}$ for 3D7 and mixed inoculation, respectively (half-distance $\approx$ $0.69 \alpha$ in 1D). Thus, experiments in controlled conditions appear to translate well into the field, but details of the models need to be considered for a sound interpretation of results. We computed the one-dimensional estimates solely to compare them to results of previous studies, although we consider the two-dimensional estimates to be more accurate.

The estimated transmission rates were $\beta_{3 \mathrm{D} 7}=7.7$ and $\beta_{\text {mix }}=$ 5.6. Parameter estimates for the strain $1 \mathrm{~A} 5$ were not realistic. We assume that these nonrealistic results were caused by issues in the fitting due to low infection success of 1A5. Strain 1A5 is known to produce fewer and smaller pycnidia than strain 3D7 on cultivar Runal in greenhouses (Stewart et al. 2018), which is further supported by our phenotypic and genotypic data in field conditions. The observed lower transmission of the mixed inoculation probably resulted from the contrasting reproductive capacities of the two strains. Thus, differences in reproductive capacities between different pathogen strains (usually assessed in controlled conditions) may have an impact on epidemic dynamics in the field and thereby influence population composition over the course of a seasonal epidemic.

Although the horizontal dispersal measurements made in previous experimental studies were useful, they are difficult to extrapolate to real field conditions for two reasons. First is that the modeling approaches did not consider the spatial extent of the source area as we did here but assumed a unique point source (Karisto et al. 2019b). Second is that a plant canopy, acting as a barrier that limits the effective dispersal distance, was not always included in the design (e.g., Brennan et al. 1985).

STB development depends on weather conditions (Henze et al. 2007). Additionally, the disease levels within the source and along the disease gradient were measured only on upper leaf layers, but dispersal occurred likely to, and possibly also from, the lower leaf layers as well, which were not included in our estimates of transmission rates. For all these reasons, the relative differences between transmission rate estimates in our experiment are informative, whereas their comparison with transmission rates measured in other studies is of limited value.

\section{Differences in dispersal}

According to our analysis, the mixture of the two strains spread further than the single strain 3D7. The difference may arise from various sources. Density-dependent effects may have flattened the disease gradient in the mixed inoculation treatment, for example, if the mixed pathogen population is more sensitive to saturation at high population densities close to the inoculated area due to cross-suppression between the two pathogen strains. In this case, the beginning of the gradient, where the population density is highest, would experience stronger saturation after mixed inoculation compared with single-strain inoculations. That would result in lower overall transmission but also a flatter gradient (i.e., longer dispersal), as the tail of the gradient would be relatively stronger after the mixed inoculation compared with the single-strain inoculations due to relaxed cross-suppression. Interestingly, these expected patterns matched with our observations in the present study.

Furthermore, sexual reproduction in the mixed inoculation treatment could result in a shallower gradient due to winddispersed ascospores, which are expected to have a much higher dispersal distance. The average latent period following infections by ascospores was shown to be longer than the latent period associated with asexual pycnidiospores (Morais et al. 2015), but the distribution of ascospore latent periods might still be wide enough to contribute to our observed gradient (Suffert and Thompson 2018). Ultimate causes of the difference remain unknown.

\section{Sampling distances}

The measurement lines were at closest $20 \pm 5 \mathrm{~cm}$ from the edge of the inoculated area. Measuring the disease intensity closer to the source and even inside the source might have improved the estimates, as the differences between the gradients are more pronounced close to the source. However, closer to source, the reliability of measurements may suffer from saturation and from dispersal via direct contact (Fitt et al. 1989).

We also measured the disease inside the inoculation area, but those data points were excluded from fitting because our aim was to analyze only the newly spread infection and capture the primary disease gradients. The increase of disease intensity in the source area from $t_{0}$ to $t_{1}$ was not only due to secondary infections but likely also from latent infections that can become symptomatic after an extended period (Karisto et al. 2019a). An additional reason for excluding data at $\mathrm{x}_{0}$ is that saturation effects are expected to be strongest there.

\section{Spatially explicit modeling}

We used spatially explicit modeling to account for the spatial extent of the source. This allowed us to parameterize the dispersal kernels, without needing to assume a point-like source. Using considerably large sources, we created sufficiently strong, measurable disease gradients in two out of three treatments. Further extension of the source might have created a sufficiently strong gradient for the strain 1A5 too. Overall, using an extended source in combination with spatially explicit modeling provides a major improvement for measuring dispersal over small scales.

\section{Statistical advances}

The bootstrapping methods allowed us to test for differences in our parameter estimates and the associated uncertainties in a robust manner without making any assumptions about underlying distributions. Computationally intensive bootstrapping with a large number of replicates $(100,000)$ is possible using modern computing resources. Nonparametric bootstrapping is a useful alternative to standard parametric tests, which are often used in biology even when their assumptions are violated. Moreover, we adopted a two-dimensional hypothesis test based on a kernel density estimate of the bootstrap parameter distribution. This allowed us conduct joint testing for differences in two parameters simultaneously, which is likely to be more robust than the combination of two one-dimensional tests for each of the parameters separately. This is demonstrated in the example where a combination of one-dimensional tests would fail to reject the null hypothesis (Fig. 3A). The provided source code and raw data will facilitate the application of these methods to other contexts.

\section{Implications for epidemiology and control}

According to our estimates, the median dispersal distance of new infection from a focal source was around $30 \mathrm{~cm}$. However, the limit of $95 \%$ of new infections would extend up to $1 \mathrm{~m}$ (equation A3; Karisto et al. 2019b). In the second dispersal step, not only will the infection spread further outward, but the secondary spread within the area covered by the first step would likely cause significant damage (Shaw and Royle 1993), and this is when a clearly visible focus of the disease can be seen (Zadoks and van 
den Bosch 1994). Thus, when one observes a visible focus in the canopy, the spread has likely already happened at least one step further, considering latent infections. This should be considered when aiming for spatially targeted control of crop diseases in the context of precision agriculture (e.g., based on aerial detection and quantification of symptoms; Anderegg et al. 2019; Yu et al. 2018). Safety margins around a disease focus depend on the expected dispersal distance of the pathogen, which highlights the importance of accurate characterization of plant pathogen dispersal in field conditions.

We note that the presented data consist of only a single time point in a single geographic location, although thoroughly replicated within that location. Our results do match well with the previous experimental outcomes in controlled conditions, but the degree to which they can be generalized is somewhat limited. In different weather conditions (especially more rainy and thus more conducive to disease) and on other wheat genotypes (those more or less susceptible to STB or representing other canopy architectures), dispersal distances are likely different, but we cannot quantify these differences based on the data we acquired here.

\section{Outlook}

Natural populations of $Z$. tritici are extremely diverse, both phenotypically and genetically (Hartmann et al. 2017; Karisto et al. 2018). Hence, it would be interesting to conduct similar measurements in a number of other $Z$. tritici strains under various conditions in the field to characterize the diversity of dispersal and transmission processes. The methodology developed here can also be applied to other plant pathosystems. We hope that our study, with the available raw data and source code of the analyses, will inspire further measurements of plant pathogen dispersal.

\section{ACKNOWLEDGMENTS}

The Genetic Diversity Centre at ETH Zurich helped with the genetic analysis. PK and AM thank Andreas Hund and Hansueli Zellweger for maintenance of the field experiment, Bruce McDonald for helpful discussions, and Marcello Zala for support in preparing inoculations and other laboratory work. AM is grateful to Jonathan Levine for his advice on designing the experiment. PK is grateful to Fanny Hartmann for help in designing PCR primers. FS thanks Christophe Montagnier, Sandrine Gélisse, and Nicolas Lecutier for maintenance of the similar field experiment prepared at the facilities of INRAE Bioger in Thiverval-Grignon, France.

\section{LITERATURE CITED}

Anderegg, J., Hund, A., Karisto, P., and Mikaberidze, A. 2019. In-field detection and quantification of Septoria tritici blotch in diverse wheat germplasm using spectral-temporal features. Front. Plant Sci. 10:1355.

Bannon, F., and Cooke, B. 1998. Studies on dispersal of Septoria tritici pycnidiospores in wheat-clover intercrops. Plant Pathol. 47:49-56.

Ben M'Barek, S., Karisto, P., Abdedayem, W., Laribi, M., Fakhfakh, M., Kouki, H., Mikaberidze, A., and Yahyaoui, A. 2020. Improved control of Septoria tritici blotch in durum wheat using cultivar mixtures. Plant Pathol. 69:1655-1665.

Brennan, R., Fitt, B. D., Taylor, G., and Colhoun, J. 1985. Dispersal of Septoria nodorum pycnidiospores by simulated raindrops in still air. J. Phytopathol. 112:281-290.

Davison, A. C., and Hinkley, D. V. 1997. Bootstrap Methods and Their Application. Cambridge University Press, Cambridge, U.K.

Dean, R., van Kan, J. A., Pretorius, Z. A., Hammond-Kosack, K. E., Di Pietro, A., Spanu, P. D., Rudd, J. J., Dickman, M., Kahmann, R., Ellis, J., and Foster, G. D. 2012. The top 10 fungal pathogens in molecular plant pathology. Mol. Plant Pathol. 13:414-430.
Djidjou-Demasse, R., Moury, B., and Fabre, F. 2017. Mosaics often outperform pyramids: Insights from a model comparing strategies for the deployment of plant resistance genes against viruses in agricultural landscapes. New Phytol. 216:239-253.

Duvivier, M., Dedeurwaerder, G., De Proft, M., Moreau, J.-M., and Legrève, A. 2013. Real-time PCR quantification and spatio-temporal distribution of airborne inoculum of Mycosphaerella graminicola in Belgium. Eur. J. Plant Pathol. 137:325-341.

Fitt, B. D., McCartney, H., and Walklate, P. 1989. The role of rain in dispersal of pathogen inoculum. Annu. Rev. Phytopathol. 27:241-270.

Fitt, B. D. L., Gregory, P. H., Todd, A. D., McCartney, H. A., and Macdonald, O. C. 1987. Spore dispersal and plant disease gradients; a comparison between two empirical models. J. Phytopathol. 118:227-242.

Gregory, P. 1968. Interpreting plant disease dispersal gradients. Annu. Rev. Phytopathol. 6:189-212.

Hartmann, F. E., Sánchez-Vallet, A., McDonald, B. A., and Croll, D. 2017. A fungal wheat pathogen evolved host specialization by extensive chromosomal rearrangements. ISME J. 11:1189-1204.

Heald, F. 1913. The address of the president for 1912: The dissemination of fungi causing disease. Trans. Am. Microsc. Soc. 32:5-29.

Henze, M., Beyer, M., Klink, H., and Verreet, J.-A. 2007. Characterizing meteorological scenarios favorable for Septoria tritici infections in wheat and estimation of latent periods. Plant Dis. 91:1445-1449.

Johansson, R., Strålfors, P., and Cedersund, G. 2014. Combining test statistics and models in bootstrapped model rejection: It is a balancing act. BMC Syst. Biol. 8:46.

Jørgensen, L. N., Hovmøller, M. S., Hansen, J. G., Lassen, P., Clark, B., Bayles, R., Rodemann, B., Flath, K., Jahn, M., Goral, T., Czembor, J. J., Cheyron, P., Maumene, C., Pope, C. D., Ban, R., Nielsen, G. C., and Berg, G. 2014. IPM strategies and their dilemmas including an introduction to www.eurowheat.org. J. Integr. Agric. 13:265-281.

Karisto, P., Dora, S., and Mikaberidze, A. 2019a. Measurement of infection efficiency of a major wheat pathogen using time-resolved imaging of disease progress. Plant Pathol. 68:163-172.

Karisto, P., Hund, A., Yu, K., Anderegg, J., Walter, A., Mascher, F., McDonald, B. A., and Mikaberidze, A. 2018. Ranking quantitative resistance to Septoria tritici blotch in elite wheat cultivars using automated image analysis. Phytopathology 108:568-581.

Karisto, P., Suffert, F., and Mikaberidze, A. 2019b. Spatially-explicit modeling improves empirical characterization of dispersal: Theory and a case study. bioRxiv 789156

Kirchgessner, N., Liebisch, F., Yu, K., Pfeifer, J., Friedli, M., Hund, A., and Walter, A. 2017. The ETH field phenotyping platform FIP: A cablesuspended multi-sensor system. Funct. Plant Biol. 44:154-168.

Klein, E. K., Bontemps, A., and Oddou-Muratorio, S. 2013. Seed dispersal kernels estimated from genotypes of established seedlings: Does densitydependent mortality matter? Methods Ecol. Evol. 4:1059-1069.

Lovell, D., Hunter, T., Powers, S., Parker, S., and van den Bosch, F. 2004a Effect of temperature on latent period of Septoria leaf blotch on winter wheat under outdoor conditions. Plant Pathol. 53:170-181.

Lovell, D., Parker, S., Hunter, T., Royle, D., and Coker, R. 1997. Influence of crop growth and structure on the risk of epidemics by Mycosphaerella graminicola (Septoria tritici) in winter wheat. Plant Pathol. 46: 126-138.

Lovell, D., Parker, S., Hunter, T., Welham, S., and Nichols, A. 2004b. Position of inoculum in the canopy affects the risk of Septoria tritici blotch epidemics in winter wheat. Plant Pathol. 53:11-21.

Madden, L. V., Hughes, G., and van den Bosch, F. 2007. The Study of Plant Disease Epidemics. American Phytopathological Society (APS Press), St. Paul, MN

McCartney, H., Fitt, B. D., and West, J. S. 2006. Dispersal of foliar plant pathogens: Mechanisms, gradients and spatial patterns. Pages 159-192 in: The Epidemiology of Plant Diseases. Cooke, B. M., Jones, D. G., and Kaye, B. eds. Springer, Dordrecht, The Netherlands.

Mikaberidze, A., McDonald, B. A., and Bonhoeffer, S. 2014. Can high-risk fungicides be used in mixtures without selecting for fungicide resistance? Phytopathology 104:324-331.

Mikaberidze, A., Paveley, N. D., Bonhoeffer, S., and van den Bosch, F 2017. Emergence of fungicide resistance: The role of fungicide dose. Phytopathology 107:1-16.

Morais, D., Laval, V., Sache, I., and Suffert, F. 2015. Comparative pathogenicity of sexual and asexual spores of Zymoseptoria tritici (Septoria tritici blotch) on wheat leaves. Plant Pathol. 64:1429-1439.

Morais, D., Sache, I., Suffert, F., and Laval, V. 2016. Is the onset of Septoria tritici blotch epidemics related to the local pool of ascospores? Plant Pathol. 65:250-260.

Mundt, C., and Browning, J. 1985. Development of crown rust epidemics in genetically diverse oat populations: Effect of genotype unit area. Phytopathology 75:607-610. 
Nathan, R., Klein, E., Robledo-Arnuncio, J. J., and Revilla, E. 2012. Dispersal kernels: Review. Pages 187-210 in: Dispersal Ecology and Evolution. Clobert, J., Baguette, M., Benton, T. G., and Bullock, J. M. eds. Oxford University Press, Oxford, U.K.

Newton, A., Begg, G., and Swanston, J. 2009. Deployment of diversity for enhanced crop function. Ann. Appl. Biol. 154:309-322.

Newton, A., and Guy, D. 2011. Scale and spatial structure effects on the outcome of barley cultivar mixture trials for disease control. Field Crops Res. 123:74-79.

Newville, M., Stensitzki, T., Allen, D. B., and Ingargiola, A. 2014. LMFIT: Non-linear least-square minimization and curve-fitting for python. Zenodo 598352.

Orellana-Torrejon, C., Vidal, T., Boixel, A.-L., Gélisse, S., Saint-Jean, S., and Suffert, F. 2021. Annual dynamics of Zymoseptoria tritici populations in wheat cultivar mixtures: A compromise between the efficiency and durability of a recently broken-down resistance gene? bioRxiv 2021.04.23.441180.

Robert, C., Garin, G., Abichou, M., Houlès, V., Pradal, C., and Fournier, C. 2018. Plant architecture and foliar senescence impact the race between wheat growth and Zymoseptoria tritici epidemics. Ann. Bot. 121:975-989.

Saint-Jean, S., Chelle, M., and Huber, L. 2004. Modelling water transfer by rain-splash in a 3D canopy using Monte Carlo integration. Agric. For. Meteorol. 121:183-196.

Sapoukhina, N., Tyutyunov, Y., Sache, I., and Arditi, R. 2010. Spatially mixed crops to control the stratified dispersal of airborne fungal diseases. Ecol. Modell. 221:2793-2800.

Shaw, M. 1987. Assessment of upward movement of rain splash using a fluorescent tracer method and its application to the epidemiology of cereal pathogens. Plant Pathol. 36:201-213.

Shaw, M. 1990. Effects of temperature, leaf wetness and cultivar on the latent period of Mycosphaerella graminicola on winter wheat. Plant Pathol. 39:255-268.

Shaw, M., and Royle, D. 1993. Factors determining the severity of epidemics of Mycosphaerella graminicola (Septoria tritici) on winter wheat in the UK. Plant Pathol. 42:882-899.

Stewart, E. L., Croll, D., Lendenmann, M. H., Sanchez-Vallet, A., Hartmann, F. E., Palma-Guerrero, J., Ma, X., and Mcdonald, B. A. 2018. Quantitative trait locus mapping reveals complex genetic architecture of quantitative virulence in the wheat pathogen Zymoseptoria tritici. Mol. Plant Pathol. 19:201-216.

Stewart, E. L., Hagerty, C. H., Mikaberidze, A., Mundt, C. C., Zhong, Z., and McDonald, B. A. 2016. An improved method for measuring quantitative resistance to the wheat pathogen Zymoseptoria tritici using high-throughput automated image analysis. Phytopathology 106: 782-788.
Suffert, F., Delestre, G., and Gélisse, S. 2018. Sexual reproduction in the fungal foliar pathogen Zymoseptoria tritici is driven by antagonistic density dependence mechanisms. Microb. Ecol. 77:1-14.

Suffert, F., and Thompson, R. N. 2018. Some reasons why the latent period should not always be considered constant over the course of a plant disease epidemic. Plant Pathol. 67:1831-1840.

Terpilowski, M., 2018. Scikit-posthocs. https://scikit-posthocs.readthedocs.

Vidal, T., Gigot, C., de Vallavieille-Pope, C., Huber, L., and Saint-Jean, S. 2018. Contrasting plant height can improve the control of rain-borne diseases in wheat cultivar mixture: modelling splash dispersal in 3-D canopies. Ann. Bot. 121:1299-1308.

Vidal, T., Lusley, P., Leconte, M., De Vallavieille-Pope, C., Huber, L., and Saint-Jean, S. 2017. Cultivar architecture modulates spore dispersal by rain splash: A new perspective to reduce disease progression in cultivar mixtures. PLoS One 12:e0187788.

Virtanen, P., Gommers, R., Oliphant, T. E., Haberland, M., Reddy, T., Cournapeau, D., Burovski, E., Peterson, P., Weckesser, W., Bright, J., van der Walt, S. J., Brett, M., Wilson, J., Jarrod Millman, K., Mayorov, N., Nelson, A. R. J., Jones, E., Kern, R., Larson, E., Carey, C., Polat, İ., Feng, Y., Moore, E. W., VanderPlas, J., Laxalde, D., Perktold, J., Cimrman, R., Henriksen, I., Quintero, E. A., Harris, C. R., Archibald, A. M., Ribeiro, A. H., Pedregosa, F., van Mulbregt, P., and SciPy 1. 0 Contributors. 2020. SciPy 1.0: Fundamental algorithms for scientific computing in Python. Nat. Methods 17:261-272.

Willocquet, L., Savary, S., McDonald, B. A., and Mikaberidze, A. 2020. A polyetic modelling framework for plant disease emergence. Plant Pathol. 69:1630-1643.

Yu, K., Anderegg, J., Mikaberidze, A., Karisto, P., Mascher, F., McDonald, B. A., Walter, A., and Hund, A. 2018. Hyperspectral canopy sensing of wheat Septoria tritici blotch disease. Front. Plant Sci. 9:1195.

Zadoks, J., and van den Bosch, F. 1994. On the spread of plant disease: A theory on foci. Annu. Rev. Phytopathol. 32:503-521.

Zadoks, J. C., Chang, T. T., and Konzak, C. F. 1974. A decimal code for the growth stages of cereals. Weed Res. 14:415-421.

Zhan, J., Kema, G. H., Waalwijk, C., and McDonald, B. A. 2002. Distribution of mating type alleles in the wheat pathogen Mycosphaerella graminicola over spatial scales from lesions to continents. Fungal Genet. Biol. 36: 128-136.

Zhan, J., Mundt, C., and McDonald, B. 1998. Measuring immigration and sexual reproduction in field populations of Mycosphaerella graminicola. Phytopathology 88:1330-1337.

Zhan, J., Mundt, C., and McDonald, B. 2000. Estimation of rates of recombination and migration in populations of plant pathogens - A reply. Phytopathology 90:324-326. 